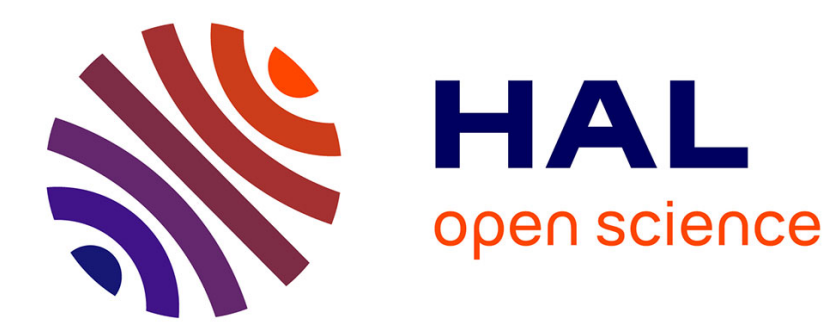

\title{
THE STRUCTURE OF CHALCOPYRITE SEMICONDUCTORS
}

D. Weaire, J. Noolandi

\section{To cite this version:}

D. Weaire, J. Noolandi. THE STRUCTURE OF CHALCOPYRITE SEMICONDUCTORS. Journal de Physique Colloques, 1975, 36 (C3), pp.C3-27-C3-29. 10.1051/jphyscol:1975305 . jpa-00216275

\section{HAL Id: jpa-00216275 https://hal.science/jpa-00216275}

Submitted on 1 Jan 1975

HAL is a multi-disciplinary open access archive for the deposit and dissemination of scientific research documents, whether they are published or not. The documents may come from teaching and research institutions in France or abroad, or from public or private research centers.
L'archive ouverte pluridisciplinaire HAL, est destinée au dépôt et à la diffusion de documents scientifiques de niveau recherche, publiés ou non, émanant des établissements d'enseignement et de recherche français ou étrangers, des laboratoires publics ou privés. 


\title{
THE STRUCTURE OF CHALCOPYRITE SEMICONDUCTORS
}

\author{
D. WEAIRE \\ Department of Physics, Heriot-Watt University, Edinburgh, U. K. \\ J. NOOLANDI \\ Xerox Research Center of Canada, Mississauga, Ontario, Canada
}

\begin{abstract}
Résumé. - Diverses tentatives faites pour comprendre la structure des semiconducteurs de type chalcopyrite par la considération de l'électronégativité des constituants sont passées en revue. Des mesures récentes de la variation thermique du rapport $q / a$ des paramètres sont également examinées.

Abstract. - We review attempts to achieve an understanding of the structure of chalcopyrite semiconductors by relating it to the electronegativity of the constituents. Recent measurements of the temperature dependence of axial ratio are also examined.
\end{abstract}

1. Introduction. - In this paper we shall discuss various questions relating to the structure of chalcopyrite semiconductors. We first consider the type of ordering found in this structure $(\$ 2)$ and then review recent attempts to set up suitable semi-empirical models for the variation of axial ratio among chalcopyrite compounds $(\S 3)$. Finally we comment on the temperature dependence of axial ratio $(\S 4)$.

2. Ordering in the chalcopyrite structure. - The chalcopyrite structure, which is found for many compounds of composition I-III-IV ${ }_{2}$ or II-IV-V 2 , may be regarded as arising from the ordered distribution of the three constituents on the sites of the diamond cubic structure, with a small distortion due to the loss of symmetry involved. The anions occupy one fcc sublattice of the diamond cubic structure while the cations share the other one. It is at first remarkable that so many compounds (several dozen) conform to the same type of ordering, so it is appropriate to consider this question first. Since many of the compounds in question have only very small distortions it would appear that a consideration of the latter is unnecessary.

If we disregard the anion sublattice we are left with one of many possible orderings of the fcc structure for equiatomic compounds. Strangely, this type of ordering is very rare in fcc alloys, being found in only a few rather exotic cases such as UTb. Instead, the CuAuI type of ordering, in which the two species occupy alternate layers perpendicular to the $(001)$ direction, is commonly found.

In both types of ordering, two thirds of the nearest neighbours of every atom are of the other species, which is as much as can be achieved, on average, for the fcc structure.
The preference for the chalcopyrite structure is less mysterious when one considers the Madelung energy associated with the structure. Hodges (unpublished) has recently shown this to be lower for the chalcopyrite type of ordering than the other one mentioned above. That fcc metallic alloys should not choose the structure of lowest Madelung energy is perhaps reasonable, on account of screening.

3. The variation of axial ratio. - There have been various attempts $[1,2]$ to relate the observed axial ratios of chalcopyrite compounds to parameters characteristic to their elements or bonds, such as covalent and ionic radii and electronegativities. Phillips [3] attempted to correlate the axial ratio with a linear function of the three (Phillips) electronegativities involved, arriving at the formula

$$
2-c / a=-0.60 X_{A}+0.25 X_{B}+0.15 X_{C}+0.01 \text {, }
$$

where $A, B$ and $C$ denote respectively the first, second and third constituents of a I-III-VI $I_{2}$ or II-IV-V compound and $X$ denotes electronegativity. This formula was only moderately successful [3].

Noolandi [4] and Weaire (unpublished) noted that, for given $A$, the distortion $2-c / a$ obeys the relation

$$
2-c / a \alpha\left(X_{A}-X_{B}\right)^{2}
$$

rather accurately.

To produce a good fit to the data, it seems that one must incorporate the squared electronegativity difference (2). For example, Noolandi's formula [4] is

$$
2-c / a=\alpha R_{A}^{\beta} R_{C}^{\nu}\left(X_{A}-X_{B}\right)^{2}
$$

where $R_{A}$ and $R_{C}$ are tetrahedral radii. This particular form for the coefficient is not demanded by the data. 
Indeed, the fact that quite different values of $\beta$ and $\gamma$ were obtained for the I-III-VI $I_{2}$ and II-IV-V $V_{2}$ families suggests that this part of the formula may not be very meaningful.

Weaire was led to test such a quadratic form from a consideration of Inglesfield's pseudopotential perturbation theory of alloys, by reasoning as follows. It is tempting to consider the problem in terms of perturbation theory, starting from an average III-V or II-VI compound. The perturbation is- associated with the $A$ and $B$ atoms only, which are arranged on an fcc structure.

The analogous problem for an fcc metal, as described by Inglesfield's perturbation theory, indeed gives a contribution to the variation of axial ratio which varies as the square of the difference of $A$ and $B$ pseudopotentials, which one may associate with the difference of their electronegativities. (This identification is not a particularly compelling one when the atoms have different valences - indeed, the whole question of the meaning of electronegativity difference in such a case is rather obscure.)

It has not proved possible to set up more detailed and justifiable arguments based on perturbation theory which recognise the existence of the C-lattice and include the displacement $x$. In attempting to construct ad hoc arguments of this kind, one must also bear in mind the Abrahams-Bernstein rule [6] relating $c / a$ and $x$. It then seems extraordinarily difficult to derive a quadratic dependence of $(x-0.25)$ upon $X_{A}-X_{B}$, since a linear dependence seems inevitable for short range forces.

Suppose, for instance, we assume that the chalcopyrite compound can be treated as a II-VI or III-V compound perturbed by additional pair wise central $A C$ and $B C$ nearest neighbour forces, equal and opposite and of magnitude proportional to the electronegativity difference of $A$ and $B$ (which we shall call $\delta X$ ).

Define $\Delta=2.0-c / a$ and $\eta=4(x-0.25)$ as in Noolandi [4].

Expanding the total energy in powers of $\Delta$ and $\eta$, we have, up to quadratic order,

$$
U=A+B \Delta+C \eta+D \Delta \eta+E \Delta^{2}+F \eta^{2} .
$$

We must now consider the dependence of the coefficients on $\delta X$. Firstly, $B$ vanishes by symmetry. $C$ is proportional to $\delta X$, as is also $D$.

Finally, the coefficients $E$ and $F$ are finite for zero $\delta X$. It follows that the equilibrium $\Delta$ and $\eta$ are

$$
\Delta=\frac{D C}{4 E F-D^{2}}
$$

and

$$
\eta=\frac{2 E C}{4 E F-D^{2}}
$$

We then obtain $\Delta=O\left(\delta X^{2}\right)$, as hoped, but unfortu- nately $\eta=0(\delta X)$, violating the Abrahams-Bernstein rule [6], which appears to be well verified and is an essentially linear relation between $\Delta$ and $\eta$ for the range of values found in experiment $(\Delta=\eta$ to first order).

This illustrates the difficulty, so far not overcome, of extracting the observed dependence from any simple model, while also conforming to the AbrahamsBernstein rule.

A curious special case which deserves more attention, is that of the compounds for which the axial ratio is equal to its ideal value 2.00 to within experimental error. This seems to happen too often to be a mere accident and yet (as has been insufficiently emphasised) there is no known theoretical reason for it. For example, Phillips' formula (1) gives $\Delta=-0.013$ for $\mathrm{ZnSnAs}_{2}$, while Noolandi's formula (3) gives $\Delta=0.012$. Shay and Wernick [7] list eight determination of axial ratios of $\mathrm{ZnSnSb}_{2}$ all of which obtain 2.00 or 2000 . Similar data exists for $\mathrm{CuInC} C_{2}$ compounds.

Since there is no theory or even semi-empirical scheme yet advanced offers any explanation for this, it would appear that the experimental situation should be reexamined. It is presumably unlikely that these compounds are disordered but a disordered arrangement of domains with different tetragonal axes is perhaps a possibility.

4. Temperature dependence of axial ratio. Recently, Miller et al. [8] have measured the temperature dependence of the axial ratios of a number of II-IV-V ${ }_{2}$ chalcopyrites. Earlier, Kildal [9] had measured this for $\mathrm{CdGeAs}_{2}$ and there is also a single measurement [10] for a I-III-VI 2 compound.

The results might at first be considered surprising, since the axial ratio increases with increasing temperature, i. e. the distortion increases. This might be contrasted with, say, In-T1 alloys for which the tetragonal axial ratio tends towards the cubic value at high temperatures - an example of the general rule that crystal structures become more symmetric at high temperatures. The case of chalcopyrite is different, however, in that due to the ordering the structure is still tetragonal even for $c / a=2.00$. There is therefore no a priori reason to expect $c / a$ to decrease with increasing temperature. Indeed we can argue the opposite, as follows. We assume that the AbrahamsBernstein rule [6] connecting $2-c / a$ with the internal displacement parameter $x$ holds at any temperature. Now, an examination of the chalcopyrite structure reveals that it is invariant under $x \rightarrow 1.0-x$, and $x \rightarrow-x$ so that $x=0$ and $x=0.5$ define structures of higher symmetry. Thus, if $x>0.25$ we expect that $x$ should tend in the direction of 0.5 (towards the nearest more symmetric structure) at high temperatures. This argument can, tentatively, be taken further, as follows. Consider the total energy $E$ as a function of $x$ (at absolute zero). One can fit a quartic to the conditions 
$\frac{\mathrm{d} E}{\mathrm{~d} x}=0$ at $x=0, \quad x=0.5, \quad x=0.25(1+\eta)$.

If we neglect all other degrees of freedom of the system, we expect the temperature coefficient $\mathrm{d} x / \mathrm{d} T$ at high temperatures to be proportional to the third derivative of $E$ at $x=0.25(1+\eta)$. Not surprisingly this is proportional to $\eta$, the (low temperature) distortion. This leads to the expectation that $\eta^{-1} \frac{\mathrm{d} \eta}{\mathrm{d} T}$ or $\Delta^{-1}$ $\frac{\mathrm{d} \Delta}{\mathrm{d} T}$ should be approximately invariant with respect to the choice of constituents within either the I-III-VI $I_{2}$ or II-IV-V $V_{2}$ families. This is a rather unsatisfactory argument when examined in detail. Its principal merit is that it seems to work ! (See Fig. 1). (It was, incidentally,

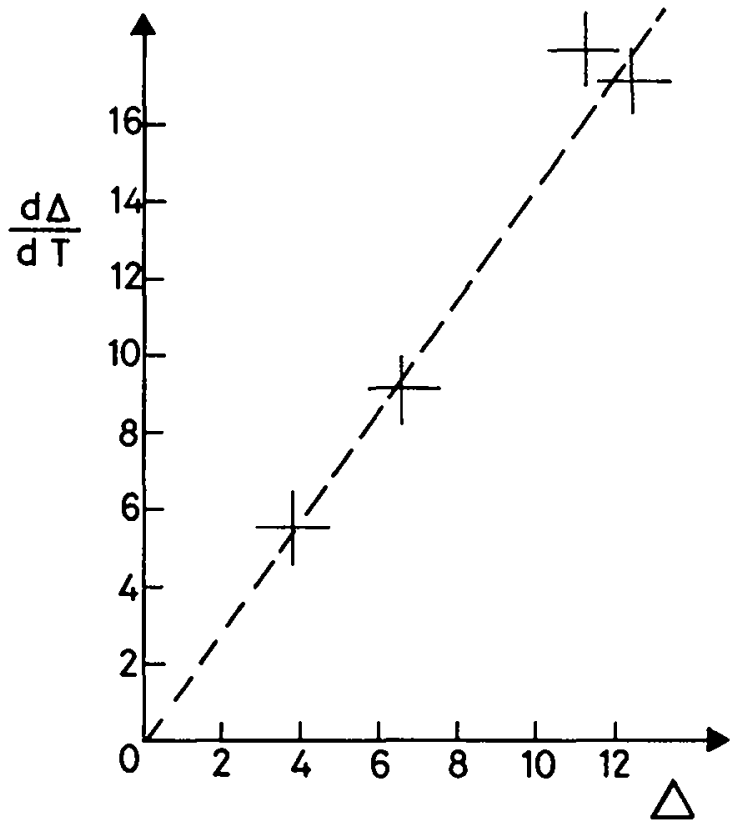

FiG. 1. - The temperature dependence of $\Delta=2.0-c / a$ is plotted against $\Delta$ for four II-IV-V $\mathrm{V}_{2}$ compounds, according to the data of reference [9] and preliminary data of A. Miller et al.

(see ref. $[8,11]$ ) [Units : $\left.\Delta, 10^{-2} ; \mathrm{d} \Delta / \mathrm{d} T, 10^{-6} \mathrm{~K}^{-1}\right]$.

advanced before much of the data of figure 1 was available.) It will be interesting to see whether this relationship, which does not seem to arise naturally from the obvious bond model [8] withstands further experimental test, and whether it can be derived from any other (and perhaps more satisfactory) point of view.

One possibility is the following. If we expand the free energy as a function of $\Delta$ (minimising $F$ with respect to $x$ for each $\Delta$ ) we have

$$
F=a+b \Delta+c \Delta^{2} \ldots
$$

and the equilibrium $\Delta$ is $-\frac{1}{2} b / c$. It follows that

$$
\Delta^{-1} \Delta^{\prime} \simeq b^{-1} b^{\prime}-c^{-1} c^{\prime}
$$

where primes denote temperature derivatives.

If the second term (which is essentially just the logarithmic derivative of an elastic constant) is dominant one might indeed expect (9) to be reasonably constant. This could come about if, for instance, $b$ had large contributions from long range forces. It is suggested that the temperature dependence of the appropriate elastic constant be measured and compared with $\Delta^{-1} \Delta^{\prime}$.

5. Conclusions, - Although interesting trends abound, the structures of chalcopyrite semiconductors are still not well understood. A knowledge of elastic constants, particularly $\frac{1}{2}\left(c_{11}-c_{12}\right)$, should help greatly in this regard and it is worthy of note that such measurements have just begun to appear $[12,13]$. It would, in particular, be interesting to compare the temperature dependence of this elastic constant with that of the axial ratio, since they are clearly intimately related. Other areas which invite further experimental investigation include the special case of the zero-distortion compounds.

The defect chalcopyrites in which ordered arrangement of vacancies is incorporated in the structure, also provide a challenge to the theorist although data seems to be scarcer and less reliable. The semi-empiricist who sets out to correlate this data with electronegativities will encounter an interesting question - what is the electronegativity of a vacancy ? When compared with the ordinary chalcopyrites the remarkable thing about these is the dominant dependence of axial ratio on the $\mathrm{C}$ cation. This remains to be explained.

Acknowledgment. - We wish to thank A. Miller for invaluable advice regarding the available data on structural properties of chalcopyrites, and C. Hodges for helpful conversations.

References

[1] Masumoto, K., Isomura, S., Goto, W., J. Phys. Chem. Solids 27 (1966) 1939.

[2] Goryunova, N. A., Grigor'era, V. S., Kradinova, L. V. and Prochukhan, V. D., Chemical Bonds in Solids (Consultants Bureau, New York) 1972, Vol. 4, p. 86.

[3] Phillips, J. C., J. Phys, Chem. Solids 35 (1974) 1205.

[4] Noolandi, J., Phys. Rev. 10 (1974) 2490.

[5] Inglesfield, J. E., J. Phys. C 2 (1969) 1285; J. Phys. C 2 (1969) 1293, Acta Metall. 17 (1969) 1395.

[6] Abrahams, S. C. and Bernstein, J. L., J. Chem. Phys. 55 (1971) 796.
[7] Shay, J. L. and WeRniCK, J. H., «Ternary Chalcopyrite Semiconductors $\gg$, to be published (1975).

[8] Miller, A., to be published (1974).

[9] KILdal, H., Ph. D. Thesis, Stanford University, Technical Report AFML TR 72-277, Wright Patterson AFB, Ohio, U.S. A.

[10] KorczaK, P. and StafF, C. B., J. Cryst. Growth 24/25 (1974) 386.

[11] Miller, A., J. Physique Colloq. 36 (1975) C 3.

[12] Grimsditch, M. H. and Holah, G. D., to be published (1975)

[13] Holah, G. D., J. Physique Colloq. 36 (1975) C 3-185. 